\title{
Fuzzy sensor aggregation: application to comfort measurement
}

\author{
Eric BENOIT, Gilles MAURIS, Laurent FOULLOY \\ LAMII/CESALP \\ Université de Savoie \\ B.P. 806, 74016 Annecy cedex
}

\begin{abstract}
This paper focuses on the acquisition of abstract information, i.e. information which are not analytically related to conventional physical quantities as for example the comfort. In these complex cases, we propose to use fuzzy sensors which compute and report linguistic assessment of numerical acquired values. Two methods are proposed to realize the aggregation from basic measurements. The first one performs the combination of the relevant features by means of a rule based description of the relations between them. With the second one, the aggregation is realised through an interpolation mechanism that make a fuzzy partition of the numeric multi-dimensional space of the basic features.
\end{abstract}

\section{Introduction}

When attempting to qualify complex phenomena, especially those related to human perception, one is often led to use words of the natural language [1], [2]. This linguistic description is less precise than the numerical one obtained through usual sensors. This description is also subjective because it depends on the observer.

Several advantages may be found in such an approach. In particular, the linguistic description is easily understood by human beings even if the concepts are abstract or if the context is changing. For example, everybody can figure out what is the concept of danger and can qualify it even if the context is unknown. Everybody is also able to classify colours without having an explicit knowledge of the origin of colours and of the perception mechanisms. Even for simple problems such as temperature measurements, human can easily integrate a priory knowledge. Imagine two people, one living close to the north pole and the other living close to the equator, speaking of a comfortable outdoor temperature in their respective countries. they are both able to abstract the concept of comfort even if the related temperatures are not the same. The representation of measurements by means of linguistic scales provides abstract information which can be integrated in decision, diagnosis or control especially with systems using symbolic coding.

Integrating capabilities of symbolic representation directly at the sensor level has led to the concept of symbolic sensor [3], [4]. The numeric-symbolic conversion has been studied in particular when the symbols of the linguistic scale have their meaning represented by means of fuzzy subsets. We have proposed to call these new sensors fuzzy symbolic sensors or simply fuzzy sensors [5], [6].

Two ways could be considered in order to obtain a linguistic description of properties or attributes from the features linked to them: defining the linguistic description by means of a rule formalism that involves a linguistic description of every feature returned by the corresponding fuzzy sensor or building the linguistic description directly on the numerical product space of the independent features by means of an interpolation method. After having recalled our formalism of the numeric-linguistic conversion, these two strategies will be investigated in this paper and then applied to a linguistic description of comfort.

\section{The numeric to linguistic conversion}

To perform a symbolic measurement, it is necessary to clearly specify the relation between symbols and numbers. Let $\mathcal{E}$ be the set of all possible measurements. Let $\mathcal{L}(\mathcal{E})$ be the set of symbols associated to this universe. Denote $\mathcal{P}(\mathcal{E})$ the set of subsets of $\mathcal{E}$. An injective mapping, called a meaning and denoted $\tau: \mathcal{L}(\mathcal{E}) \rightarrow \mathcal{P}(\mathcal{E})$ associates any symbol with a subset of measurements. Injectivity means that two symbols with the same meaning should be considered as identical. Symbolic measurement is now obtained from a mapping, called a 
description and denoted $\mathrm{l}: \mathcal{E} \rightarrow \mathcal{P}(\mathcal{L}(\mathcal{E})$ ). It associates any measurement with a subset of the symbolic set $\mathcal{L}(\mathcal{E})$.

There is a fundamental relation between description and meaning. If a symbol belongs to the description of a measurement, then the measurement belongs to the meaning of the symbol, i.e:

$$
L_{l} \in \mathrm{l}(x) \Leftrightarrow x \in \tau\left(L_{l}\right)
$$

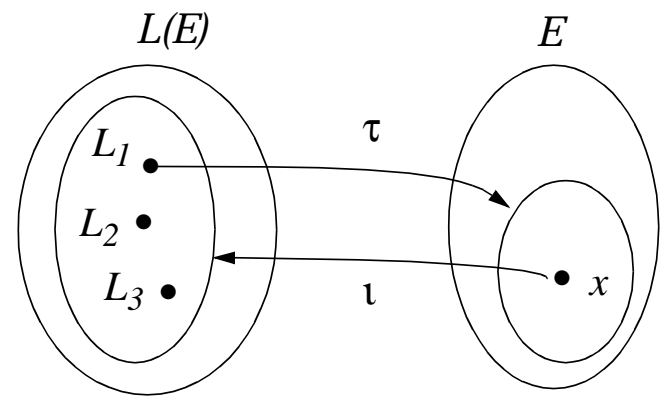

Fig. 1 : Relation between the description and the meaning

Here is an example for a sensor that returns the ambient temperature. Let the measurement set be $T=\left[\begin{array}{llll}0 & { }^{\circ} \mathrm{C}, & 40 & { }^{\circ} \mathrm{C}\end{array}\right]$ and the symbolic set be $\mathcal{L}(T)=\{$ cold, cool, mild, warm, hot $\}$ The following figure shows an example of symbol meanings:

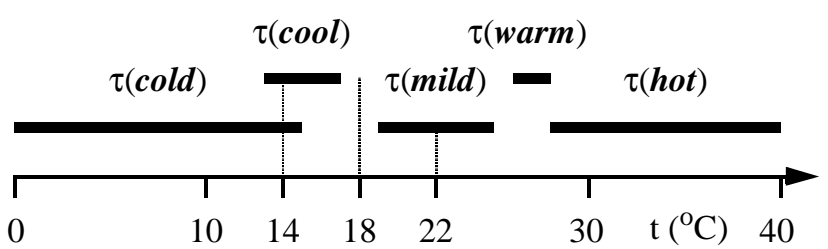

Fig. 2 : Meanings of items of $\mathcal{L}(T)$

The descriptions of measurements comes directly from the definitions. For example:

$$
\begin{gathered}
\mathrm{l}\left(22^{\circ} \mathrm{C}\right)=\{\text { mild }\} \\
\mathrm{l}\left(14{ }^{\circ} \mathrm{C}\right)=\{\text { cold }, \text { cool }\} \\
\mathfrak{l}\left(18^{\circ} \mathrm{C}\right)=\varnothing
\end{gathered}
$$

In the general case, the description of a measure can contain any number of symbols. In a natural language, a measure is usually described by only one symbol. To apply this condition we impose the set of the meanings to be a partition of the measurement set (fig. 3).

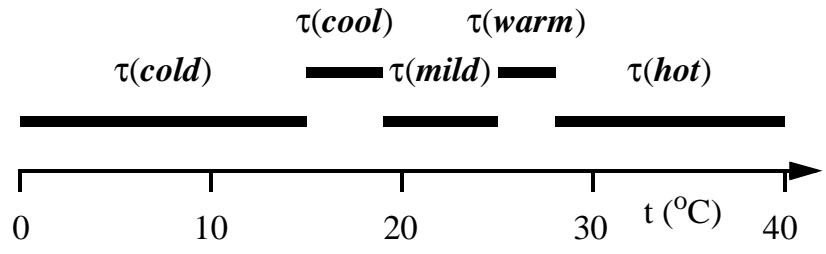

Fig. 3 : Meanings satisfying the partition

\section{The fuzzy numeric-symbolic conversion}

The previous approach, based on subsets, obviously leads to sharp transitions in the sensor response. Fuzzy subset theory, developed by Zadeh, provides a nice solution to this problem [7], [8]. The extension of the characteristic function of a crisp subset (values in $\{0,1\}$ ) to the membership function of a fuzzy subset (values in $[0,1])$ can be used to model gradual transitions between symbols.

The fuzzy numeric to symbolic interface depends on the extension to the fuzzy case of the definitions of the previous section. So fuzzy meaning and fuzzy description have to be defined [9]. The fuzzy meaning is a mapping from the symbolic set $\mathcal{L}$ to the set of the fuzzy subsets of measurements. The fuzzy meaning of a symbol $L$ is characterized by its membership function denoted $\mu_{\tau(L)}(x)$.

In a same manner, a fuzzy description can be defined as a mapping from the measurement set into the set of the fuzzy subsets of symbols, so the fuzzy description is characterized by its membership function denoted $\mu_{\mathrm{l}(x)}(L)$.

The relation between the membership functions of a fuzzy description and the corresponding fuzzy meaning comes directly from the fundamental relation between the meaning and the description, i.e:

$$
\mu_{\imath(x)}(L)=\mu_{\tau(L)}(x)
$$

In order to conserve a link between the sensor description and the human feeling, we impose that the set of symbols meaning is a fuzzy partition of the measurement set in the sense of Bezdek [10]. Then, the triplet $\langle E, \mathcal{L}(E), \mathrm{\imath}\rangle$ is a fuzzy nominal scale [6].

$$
\Sigma_{\mathrm{a} \in \mathcal{L}(T)} \mu_{\mathrm{l}(t)}(\boldsymbol{a})=1
$$




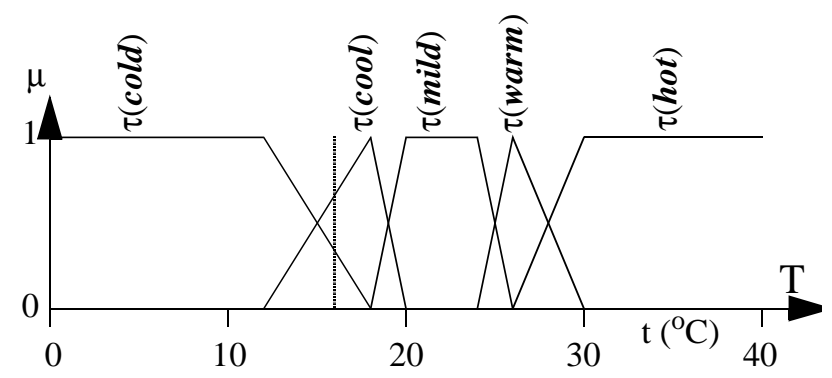

Fig. 4 : Meanings of the temperature lexical set

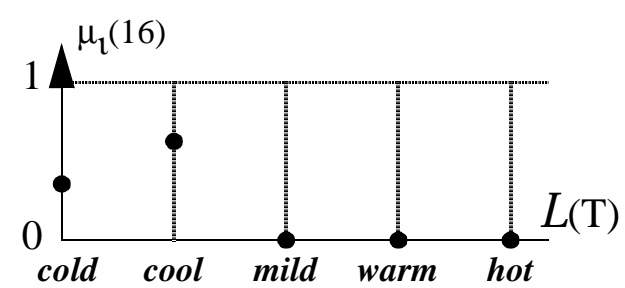

Fig. 5 : Description of the measure $16^{\circ} \mathrm{C}$

\section{Rule based symbolic aggregation}

With multi-dimensional measurements, the definition of symbolic meanings leads to a combination between basic measurements. For example, the description of comfort needs the knowledge of the relative humidity and of the temperature. Then a linguistic definition of the comfort can be modelized by a set of rules. Let us describe by this example the mechanism used to define the semantic of comfort.

The temperature and humidity measurements take their respective values in the sets denoted $T$ and $H$. The temperature is described by symbols in the set $\mathcal{L}(T)=\{$ cold, cool, mild, warm, hot $\}$. The humidity is described by symbols in the set $\mathcal{L}(H)=\{$ very_low, low, medium, high $\}$. It also assumed that the meanings of the symbols generate a partition of the respective numerical sets.

The problem is now to aggregate both measurements to obtain information about the feeling of comfort. Such a feeling could be symbolically defined as follows.

atmosphere is comfortable when : the tempera-

ture is mild and the humidity is medium

atmosphere is acceptable when : the temperature is cool or mild or warm and the humidity is low or the temperature is cool or warm and the humidity is medium

atmosphere is uncomfortable when : atmosphere is not (comfortable or acceptable)

One solution is to consider that two symbols connected by the and operator are in fact one symbol whose meaning is defined on the cartesian product of the numerical sets. For example, "the temperature is mild and humidity is medium" should be no more considered as the two expressions connected by the operator and, but as one expression such as "the temperature_humidity is mild_medium". The new symbolic variable temperature_humidity takes its value in a new set of symbols, for example denoted $\mathcal{L}(T H)$. The symbol mild_medium belongs to $\mathcal{L}(T H)$, and its meaning is defined on the cartesian product $T \times \mathrm{x} H$. Then the meaning of comfortable is defined by

$$
\begin{gathered}
\tau(\text { comfortable })=\tau(\text { mild_medium }) \\
=\tau(\text { mild }) \times \tau(\text { medium })
\end{gathered}
$$

The meanings of the or and the not operators are respectively defined by the union operator and by the negation operator.

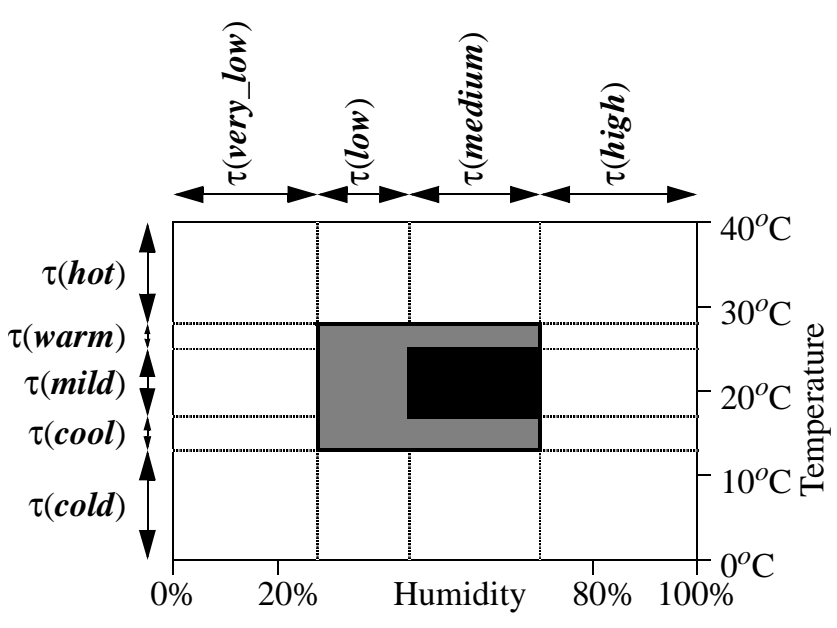

$\tau($ comfortable $) \quad \square \tau($ acceptable $) \quad \square \tau($ uncomfortable $)$

Fig. 6 : Graph representing the meanings of comfortable, acceptable and uncomfortable

\section{Rule based fuzzy sensor aggregation}

Let $t$ and $h$ be the temperature and humidity measurement. The fuzzy description of the couple $(t, h)$ can be expressed in terms of the fuzzy 
descriptions of $t$ and $h$ by means of a triangular norm $\mathrm{T}$.

$$
\mu_{\mathfrak{l}(t, h)}\left(\boldsymbol{a} \_\boldsymbol{b}\right)=\mu_{\mathfrak{l}(t)}(\boldsymbol{a}) \mathrm{T} \mu_{\mathfrak{l}(h)}(\boldsymbol{b})
$$

As a fuzzy partitioning, in the sense of Bezdek, was chosen for the temperature and the humidity, we have :

$$
\Sigma_{\mathrm{a} \in \mathcal{L}(T)} \mu_{\mathrm{l}(t)}(\boldsymbol{a})=1 \text { and } \Sigma_{\mathrm{b} \in \mathcal{L}(H)} \mu_{\mathrm{l}(h)}(\boldsymbol{b})=1
$$

Therefore, if we want to obtain a fuzzy partition for the new symbolic set $\mathcal{L}(T H)$, the triangular norm has to be distributive with respect to the addition (for example the product).

$$
\begin{gathered}
\sum_{\mathrm{a} \_\mathrm{b} \in \mathcal{L}(H T)} \mu_{\mathrm{l}(t, h)}\left(\boldsymbol{a} \_\boldsymbol{b}\right) \\
=\sum_{\mathrm{a} \in \mathcal{L}(T)} \Sigma_{\mathrm{b} \in \mathcal{L}(H)} \mu_{\mathfrak{l}(t)}(\boldsymbol{a}) \mathrm{T} \mu_{\mathfrak{l}(h)}(\boldsymbol{b}) \\
=\Sigma_{\mathrm{a} \in \mathcal{L}(T)} \mu_{\mathfrak{l}(t)}(\boldsymbol{a}) \mathrm{T} \Sigma_{\mathrm{b} \in \mathcal{L}(H)} \mu_{\mathfrak{l}(h)}(\boldsymbol{b}) \\
=\sum_{\mathrm{a} \in \mathcal{L}(T)} \mu_{\mathfrak{l}(t)}(\boldsymbol{a}) \mathrm{T} 1 \\
=\sum_{\mathrm{a} \in \mathcal{L}(T)} \mu_{\mathfrak{l}(t)}(\boldsymbol{a})=1
\end{gathered}
$$

Now, we have to define the fuzzy meaning of the or operator. Let $E$ be a set of measurements and $\mathcal{L}(E)$ its associated set of symbols. Let $L_{1}$ and $L_{2}$ be two symbols of $\mathcal{L}(E)$. Let $\perp$ be the triangular conorm which defines the fuzzy meaning of or:

$$
\begin{aligned}
& \mu_{\tau\left(L 1 \_o r \_L 2\right)}(x) \\
= & \mu_{\tau(L 1)}(x) \perp \mu_{\tau(L 2)}(x) \\
= & \mu_{1(x)}\left(L_{1}\right) \perp \mu_{1(x)}\left(L_{2}\right)
\end{aligned}
$$

Denote $\mathcal{L}^{*}(E)=\mathcal{L}(E)-\left\{L_{1}, L_{2}\right\}$, and $\mathcal{L}^{+}(E)=$ $\mathcal{L}(E) \cup\left\{L_{1} \_\right.$or_ $\left.L_{2}\right\}$

The triangular conorm must be chosen such that the meaning of the symbols of $\mathcal{L}^{+}(E)$ forms also a fuzzy partition:

$$
\begin{gathered}
\sum_{L \in \mathcal{L}(E)} \mu_{\mathrm{l}(x)}(L) \\
=\sum_{L \in \mathcal{L}^{*}(E)} \mu_{\mathrm{l}(x)}(L)+\mu_{\mathrm{l}(x)}(L 1)+\mu_{\mathrm{l}(x)}(L 2) \\
=1 \\
\sum_{L \in \mathcal{L}^{+}(E)} \mu_{\mathrm{l}(x)}(L) \\
=1
\end{gathered}
$$

Therfore we have

$$
\mu_{\mathrm{l}(x)}\left(L_{1}\right) \perp \mu_{\mathrm{l}(x)}\left(L_{2}\right)=\mu_{\mathrm{l}(x)}(L 1)+\mu_{\mathrm{l}(x)}(L 2) \leq 1
$$

Thus, the triangular conorm should verify

$$
\mathrm{x} \perp \mathrm{y}=\mathrm{x}+\mathrm{y} \text { if } \mathrm{x}+\mathrm{y} \leq 1
$$

for example $: \mathrm{x} \perp \mathrm{y}=\min (\mathrm{x}+\mathrm{y}, 1)$. This is the triangular conorm chosen to define the or operator.

The meaning of the negation operator not is defined by: $\mu_{\tau(\operatorname{not} L)}(x)=1-\mu_{\tau(L)}(x)$

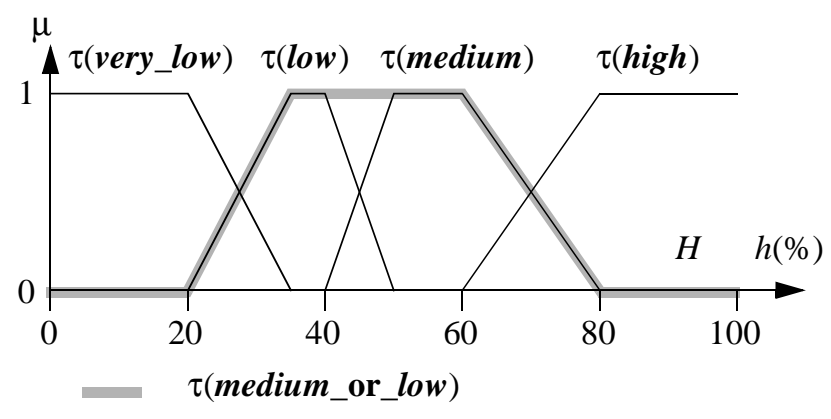

Fig. 7 : Meanings of the humidity lexical set, and meaning of a compound symbol

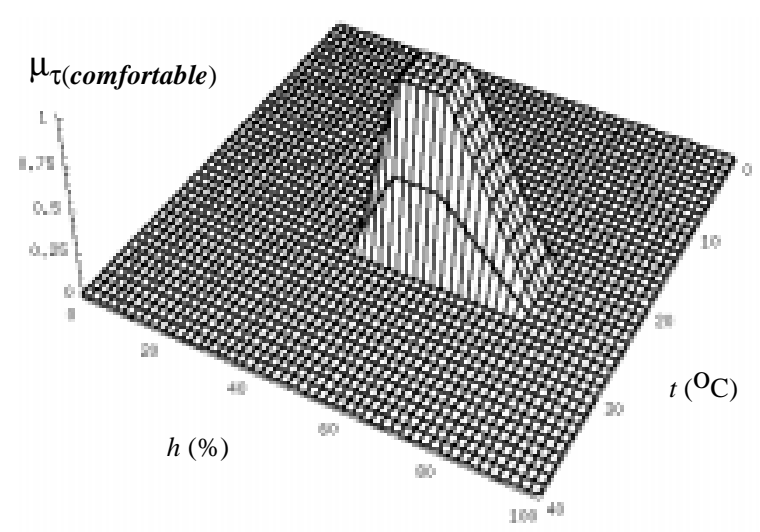

Fig. 8 : Fuzzy meaning of comfortable

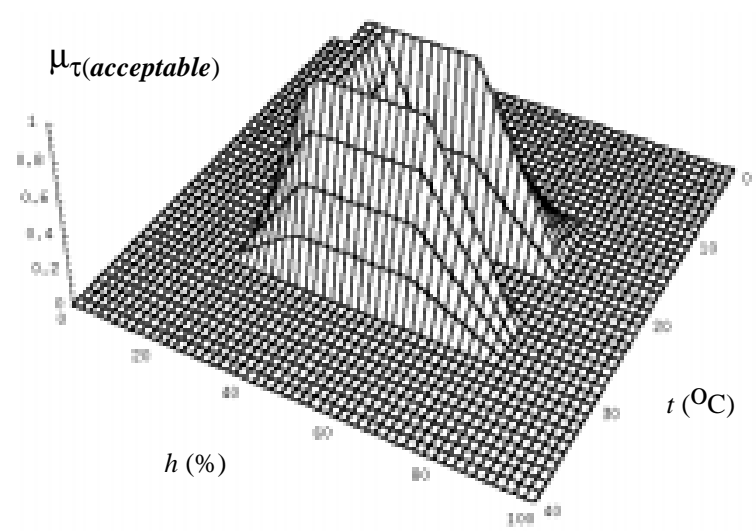

Fig. 9 : Fuzzy meaning of acceptable.

The fuzzy sensor is now able to describe the temperature and humidity for the symbol comfortable by a grade of membership which 
qualify the comfort feeling. Three results are given below for three different values of the temperature in ${ }^{\circ} \mathrm{C}$ and humidity in $\%$.

$$
\begin{gathered}
\mu_{\mathfrak{l}(23,50)}(\text { comfortable })=1 \\
\mu_{\mathfrak{l}(25,60)}(\text { comfortable })=0.5 \\
\mu_{\mathfrak{l}(5,80)}(\text { comfortable })=0
\end{gathered}
$$

\section{Multi-component numeric-symbolic conversion}

When a sensor uses several transducers, the measure is a vector of numerical values, and the measurement set is a multi-dimensional volume. An alternative way to the preceding method is to define directly the meaning of each symbol on this multi-dimensional volume. Furthermore the set of meanings has to be a fuzzy partition of the measurement set.

In this section, we consider an initial knowledge about the measurements. This knowledge is materialized by the meaning of symbols on a small subset $V$ of the measurement set. Then the measurement set is partitioned in nsimplexes with the Delaunay triangulation method. A n-simplex in a n-dimensional space is a polyhedra with $n+1$ vertices. For example, a 2simplex is a triangle and a 3 -simplex is a tetrahedron. The points used to perform the triangulation are the elements of the subset $V$.

The membership function of the meaning of each symbol is defined by piece on the $n$ simplexes. A multi-linear interpolation is used to define this function on each n-simplex. We suppose the restriction on a n-simplex of the membership function of the meaning of a symbol $s$ is :

$\mu_{\tau_{E}(s)}(v)=\mu_{\tau_{E}^{(s)}}\left(x_{1}, \ldots, x_{n}\right)=a_{1} x_{1}+\ldots+a_{n} x_{n}+a_{n+1}$

The value of this function is known for the $n+1$ vertices of the n-simplex. Indeed, the vertices are elements of the subset $V$. So the $n+1$ factors $a_{i}$ can be computed by resolving the system of $n+1$ equations with $n+1$ unknowns.

$$
A=M^{-1} B \quad B=\left[\begin{array}{c}
\mu_{\tau_{V}(s)}\left(v_{1}\right) \\
\mu_{\tau_{V}(s)}\left(v_{2}\right) \\
\ldots \\
\mu_{\tau_{V}(s)}\left(v_{n+1}\right)
\end{array}\right]
$$

$$
\Lambda=\left[\begin{array}{cccc}
x_{11} & \ldots & x_{1 n} & 1 \\
\ldots & \ldots & \ldots & \ldots \\
x_{n+1} & \cdots & x_{n+1} & 1
\end{array}\right] \quad A=\left[\begin{array}{c}
a_{1} \\
\cdots \\
a_{n+1}
\end{array}\right.
$$

Where $v_{i}$ is the $\mathrm{i}^{\text {th }}$ vertex of the $\mathrm{n}$-simplex, and $x_{i j}$ is its $\mathrm{j}^{\text {th }}$ component.

This process is performed on each n-simplex and for each symbol. Then we have a fuzzy nominal scale defined on $\mathcal{E}$. This scale is an extension of the fuzzy nominal scale on $V$.

With this method, the knowledge needed to configure the sensor is very compact. It can be acquired during a learning phase by a communication with a system called teacher which can be a man or an expert system. During the learning phase, the teacher and the sensor analyse the same phenomenon, and the teacher gives its description to the sensor. The sensor increases its knowledge with its measure associated to the teacher description. Then it owns a crips meaning of the symbols on the subset of the measurement set. The sensor can now build the fuzzy nominal scale on the measurement set. This technique was succefully used to implement a fuzzy color sensor [11].

If we consider the example of comfort measurement, the meaning of comfortable, acceptable, uncomfortable under a subset $V$ of $T \mathrm{x} H$ are defined as follow:

$\tau_{V}($ comfortable $)=\{(20,50)\}$

$\tau_{V}($ acceptable $)=\{(26,35),(16,35),(24,65)$, $(18,65),(28,50),(15,50),(20,25),(20,80)\}$

$\tau_{V}($ uncomfortable $)=\{(0,0),(20,0),(40,0)$, $(0,100),(20,100),(40,100),(0,50),(40,50)\}$

The following figure shows the meaning of comfortable and of acceptable under $\mathrm{T} \times \mathrm{H}$. 


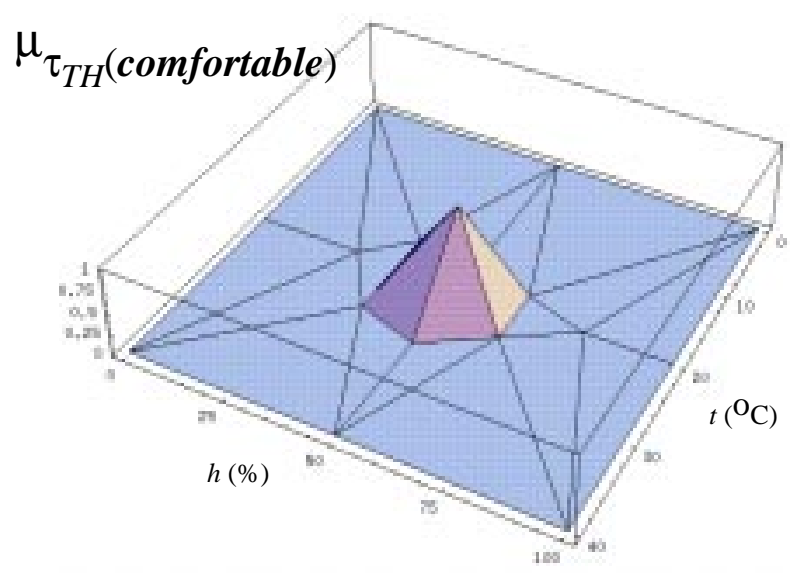

Fig. 10 : Meaning of comfortable

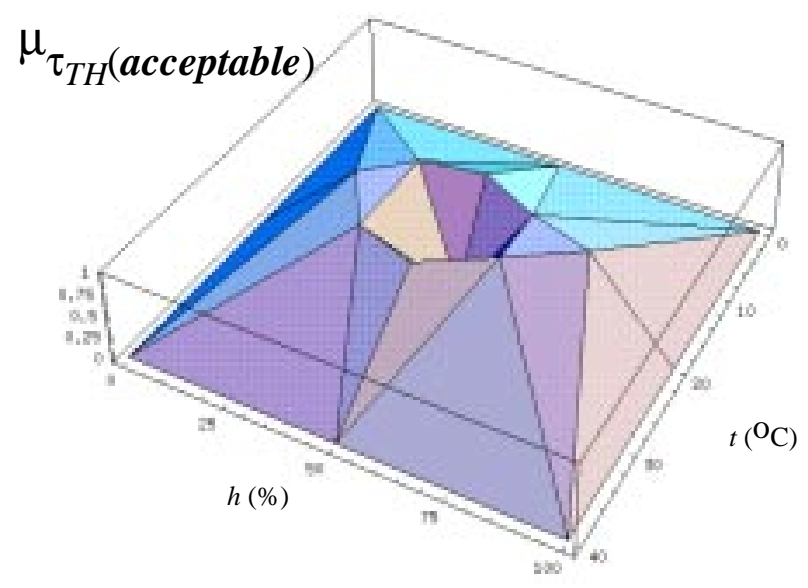

Fig. 11 : Meaning of acceptable

\section{Conclusion}

This paper has been concerned by the aggregation of complementary information using fuzzy sensors which compute and report linguistic assessments of numerical acquired values. When the aggregation cannot be represented by a numerical model, we have proposed to use a linguistic model of the aggregation or to use a set of characteristic examples associated with a fuzzy interpolative method. The rule based method is particularly efficient when the basic features are of a linguistic type as it is often the case at the reasoning level. In this approach, all the numerical operations are made at the low level (i.e. inside the sensor or the actuator). So the central unit has a reduced computation load, because it works on compact information. With the interpolative method, the computation load is higher because it works on a multidimensional numerical space. But it provides a richer structure for the output information. The first proposed method seems more adapted to complex systems that handle a lot of information and in which the perception is only one of the different considered tasks. The second proposed method seems more adapted to cases where the perception is the principal objective.

\section{References}

[1] Finkelstein L. "Representation by symbol systems as an extension of the concept of measurement", Kybernetes, Vol. 4, pp. 215-223, 1975.

[2] Zingales G., Narduzzi C., "The role of artificial intelligence in measurement.", Proc. of IMEKO TC7 int. symp. on AIMaC'91, Kyoto, Japan, 1991, pp. 212.

[3] Benoit E., Foulloy L., "Symbolic sensors : one solution to the numerical-symbolic interface", Proc. of the IMACS DSS\&QR workshop, Toulouse, France, march 1991.

[4] Benoit E., Foulloy L., "Symbolic sensors", 8th International Symposium on Artificial Inteligence based Measurement and Control (AIMaC'91), september 1991, Ritsumeikan University, Kyoto, Japan.

[5] Foulloy L., Benoit E., Mauris G., “Applications of fuzzy sensors", European Workshop on Industrial Fuzzy Control and Applications, Barcelona, Spain, April 93.

[6] Mauris G., Benoit E., Foulloy L., "Fuzzy symbolic sensors : From concept to applications", to appear in the Int. Journal of Measurement, 1994.

[7] Zadeh L. A., "Fuzzy sets", Information and control, Vol. 8, pp.338 - 353, 1965.

[8] Zadeh L. A., "The concept of a linguistic variable and its application to approximate reasoning", Information Sciences, part 1:Vol 8, No 3, pp. 199249,part 2: Vol 8,pp. 301-357, part 3:Vol 9, pp.43-80, 1975.

[9] Zadeh L.A., "Quantitative fuzzy semantics", Information Sciences, Vol. 3, 1971, pp. 159-176

[10] Bezdek J., "Pattern recognition with fuzzy objective functions”, Plenum, 1981, New-York.

[11] Benoit E., "Capteurs symboliques et capteurs flous: un nouveau pas vers l'intelligence", Thèse de doctorat de l'Université Joseph Fourier Grenoble I, January 1993. 